\title{
Pacientes con corazón univentricular sometidos a cirugía de Glenn
}

\author{
Patients with univentricular heart submitted to Glenn surgery \\ Juan L. Ramírez-Roa ${ }^{1 *}$ y Mónica I. Guzmán-Bustamante ${ }^{2}$ \\ ${ }^{1}$ Cardiología Pediátrica, Facultad de Medicina, Universidad Pontificia Bolivariana; ${ }^{2}$ Clínica Cardio VID. Medellín, Colombia
}

\section{Resumen}

Objetivo: Describir las características clínicas, quirúrgicas y posquirúrgicas de pacientes univentriculares sometidos a cirugía de Glenn en un centro de referencia cardiovascular. Método: Estudio descriptivo, retrospectivo, llevado a cabo entre enero de 2012 y diciembre de 2016, en pacientes menores de 18 años que consultaron a una clínica de cuarto nivel, con cardiopatías de fisiología univentricular, definidos por ecocardiografía institucional, presentados en junta médico-quirúrgica, operados o no como primer estadio de paliación y seguidos en el programa de ventrículo único de la institución, posterior a un cateterismo cardiaco para ser presentado en junta médica que definió la realización de cirugía de Glenn. De la historia clínica se recolectaron datos de ecocardiogramas diagnósticos, cateterismos cardiacos, descripciones quirúrgicas y evoluciones. Resultados: Se analizaron 88 pacientes univentriculares, de los cuales el 63\% eran hombres y el 36\% eran mujeres. La anatomía del ventrículo funcional univentricular derecho estuvo presente en el 38.6\% y la morfología funcional izquierda en el $61.4 \%$. Entre las características asociadas con la mortalidad se verificó que el $1.1 \%$ tuviera insuficiencia moderada de la válvula atrioventricular y que el 3.4\% tuviera insuficiencia grave de la válvula atrioventricular. El 38.6\% presentó cifras de presión pulmonar elevadas, medidas por cateterismo cardiaco, y el 46\% tenían resistencia vascular pulmonar aumentada. Se usó terapia vasopresora antes de la cirugía de Glenn en nueve pacientes; todos recibieron milrinona. La mortalidad posquirúrgica fue del 18\%. Conclusiones: Este estudio evidencia que el diagnóstico y la intervención temprana contribuyen a reducir la morbimortalidad en los pacientes con diagnóstico de corazón univentricular, puesto que sin intervención de segundo estadio de paliación sería mortal para la mayoría de ellos. Así mismo, evidencia la importancia de la implementación de un programa integral para la atención de enfermedades cardiovasculares complejas.

Palabras clave: Obstrucción del flujo ventricular externo. Cirugía torácica. Síndrome de circulación fetal persistente. Cuidados paliativos. Fenómenos fisiológicos cardiovasculares.

\section{Abstract}

Objective: To describe the clinical, surgical and post-surgical characteristics of univentricular patients undergoing Glenn's surgery in a cardiovascular reference center. Method: Descriptive, retrospective study from January 2012 to December 2016 , in patients under 18 who consulted a fourth level clinic with cardiopathies of univentricular physiology defined by institutional

\section{Correspondencia:}

*Juan L. Ramírez-Roa

E-mail: juanleandror@ hotmail.com
Fecha de recepción: 11-09-2018

Fecha de aceptación: 03-08-2020

DOI: 10.24875/RCCAR.M21000055
Disponible en internet: 29-07-2021 Rev Colomb Cardiol. 2021;28(3):289-296 www.rccardiologia.com 0120-5633 / @ 2020 Sociedad Colombiana de Cardiología y Cirugía Cardiovascular. Publicado por Permanyer. Este es un artículo open access bajo la licencia CC BY-NC-ND (http://creativecommons.org/licenses/by-nc-nd/4.0/). 
echocardiography, presented at the surgical medical board, operated or not as the first stage of palliation, followed in the single ventricle program of the institution, later performed a cardiac catheterization to be presented at the medical board that defined the performance of Glenn's surgery. From the clinical history, data of diagnostic echocardiograms, cardiac catheterizations, surgical descriptions, and evolutions were collected. Results: $A$ total of 88 univentricular patients were analyzed, of which $63 \%$ were men and $36 \%$ women. The anatomy of the right univentricular functional ventricle was present in $38.6 \%$ and $61.4 \%$ of left functional morphology. Among the characteristics associated with mortality, it was verified that $1.1 \%$ had moderate atrioventricular valve insufficiency and $3.4 \%$ had severe atrioventricular valve insufficiency in the patients who participated in the study. $38.6 \%$ had pulmonary arterial hypertension detected by cardiac catheterization and $46 \%$ had increased pulmonary vascular resistance. The use of vasopressor therapy before Glenn's surgery was present in 9 patients and of them, the whole had milrinone. The postoperative mortality was $18 \%$. Conclusions: This study shows that diagnosis and early intervention reduce morbidity and mortality in patients with a diagnosis of univentricular heart since without intervention of the second stage of palliation would be fatal for the vast majority of patients. It also demonstrates the importance of a comprehensive program for the care of complex cardiovascular pathologies.

Key words: Ventricular outflow obstruction. Thoracic surgery. Persistent fetal circulation syndrome. Palliative care. Cardiovascular physiological phenomena.

\section{Introducción}

El corazón univentricular corresponde a un tipo de cardiopatía congénita compleja del infante, que supone del $1 \%$ al $3 \%{ }^{1}$. Estos pacientes requieren un manejo cardiovascular especializado para lograr sobrevivir, ya que sin este tipo de cuidado la enfermedad es mortal.

En el año 2012, el International Nomenclature Committee for Pediatric and Congenital Heart Disease definió esta cardiopatía como un espectro de malformaciones cardiovasculares congénitas en las que la masa ventricular no puede ser dividida de manera que se tenga una bomba ventricular para la circulación sistémica y otra para la circulación pulmonar. Esto podría deberse a que tal división quirúrgica no sea anatómicamente factible 0 aconsejable ${ }^{2}$.

Por su parte, las tres fases de paliación univentricular se denominan «camino univentricular». A medida que el paciente progresa a lo largo del camino univentricular, su fisiología cardiovascular cambia. Inicialmente tendrá la fisiología de su enfermedad de base, luego pasará a la fisiología de Glenn y finalmente a la de Fontan².

El corazón univentricular tiene unos estadios de paliación definidos desde 1980: primero o paliación neonatal, segundo o derivación cavopulmonar superior bidireccional, y tercero o derivación cavopulmonar total ${ }^{3}$.

En la fisiología univentricular se cumple la mezcla completa de los retornos venosos sistémico y pulmonar, con independencia de en qué parte del corazón se combine y qué masa ventricular eyecte este retorno venoso, completamente mezclado, hacia ambas circulaciones (sistémica y pulmonar) en paralelo².
La característica fisiológica fundamental del corazón univentricular es que el ventrículo único se encuentra siempre sobrecargado, ya que responde por la circulación pulmonar y la sistémica ${ }^{4}$.

Para que este sistema hemodinámico funcione se deben cumplir dos requisitos básicos: resistencias pulmonares bajas y baja presión de fin de diástole del ventrículo sistémico ${ }^{4-6}$.

Los factores pronósticos de mortalidad en los pacientes con corazón univentricular antes de la cirugía de Glenn están definidos por artículos descriptivos ${ }^{5-9}$ : presión media en la arteria pulmonar $>18 \mathrm{mmHg}$ medida por cateterismo cardiaco (si está aumentada se denomina hipertensión pulmonar del corazón univentricular), relación flujo pulmonar/flujo sistémico, tiempo de perfusión > 70 minutos, anomalías asociadas significativas, peso $\leq 10 \mathrm{~kg}$ para cirugía de Glenn y morfología univentricular derecha.

El caso se distingue por la existencia de alteraciones en las válvulas auriculoventriculares, dadas por estenosis o atresia, que se abren sobre una cámara ventricular única, separada de este ventrículo por un pliegue muscular de una cámara rudimentaria ${ }^{10}$. De la cámara ventricular pueden nacer una arteria normal y una arteria atrésica o muy estenótica ${ }^{11}$. El cuadro clínico es sumamente variable según la edad a la cual se realice el diagnóstico, aunque habitualmente es en el periodo neonatal; lo más frecuente es que la enfermedad haga su presentación con cianosis o bajo gasto, según sea el circuito hipoplásico o atrésico ${ }^{4}$.

Al dividir la alteración según el tracto de salida afectado se encuentra que, si la alteración está en el lado derecho, su manifestación principal es falta de flujo 
pulmonar y se presentará cianosis sin dificultad respiratoria significativa. Pero si la alteración está al lado del tracto de salida del ventrículo izquierdo, su principal alteración se dará por falta de flujo sistémico y se manifestará con colapso hemodinámico y pobre perfusión del lecho vascular sistémico, hipotensión, taquipnea, choque, pulsos periféricos disminuidos, falla renal, enterocolitis y otras afectaciones de hipoflujo sistémico. ${ }^{12}$

La fisiología univentricular tiene una paliación en el periodo neonatal que se diferencia según la anatomía del ventrículo único funcional, la emergencia de los grandes vasos y las conexiones auriculoventriculares y ventriculoarteriales, entre otros.

Las cirugías para corazón derecho hipoplásico en primer estadio son la fístula Blalock-Taussig, para incrementar el flujo pulmonar y dar un flujo sistémico normal, y el cerclaje de la arteria, para disminuir el flujo pulmonar en cardiopatías con flujo pulmonar aumentado, como es el caso de la atresia tricúspide con vasos normorrelacionados con defecto septal ventricular amplio $^{4,5,13}$.

La cirugía paliativa en primer estadio para corazón izquierdo hipoplásico es la cirugía de Norwood para los pacientes con síndrome de ventrículo izquierdo hipoplásico, la cual se realiza para dar flujo sistémico por una neoaorta y dar flujo pulmonar por medio de una fístula sistémico-pulmonar o ventrículo-pulmonar ${ }^{5,14}$. Otra opción quirúrgica es la cirugía de Damus-Kaye-Stansel (DKS), la cual se utiliza cuando el tracto de salida del ventrículo izquierdo tiene flujo, pero es insuficiente. En ese caso, se secciona la arteria pulmonar y se anastomosa a la aorta nativa del paciente, con el fin de crear dos salidas hacia un vaso que emerge del corazón y dar flujo pulmonar por medio de una fístula sistémico-pulmonar ${ }^{5,15,16}$.

Posteriormente, en el segundo estadio de corazón univentricular se practica la cirugía de Glenn. La primera derivación cavopulmonar fue realizada por el Dr. Carlon en 1951 en la Universidad de Padua, y posteriormente el Dr. Glenn llevó a cabo la primera derivación exitosa de vena cava superior a la arteria pulmonar derecha (derivación Glenn clásica) en 1958 en la Universidad de Yale ${ }^{3,5}$. La cirugía de Glenn consiste en una anastomosis cavopulmonar que genera un flujo obligado del retorno de la vena cava superior a través del lecho capilar pulmonar². La derivación de Glenn sigue siendo una etapa integral en la paliación de las cardiopatías congénitas de ventrículo único. Con el tiempo, la clásica derivación de Glenn ha evolucionado de una anastomosis cavopulmonar unidireccional a una bidireccional. Esto proporcionó menos distorsión a la arteria pulmonar derecha y permitió mejorar el flujo de sangre al pulmón izquierdo ${ }^{3}$. Posteriormente, los corazones que cumplen con los criterios se llevan a cirugía de Fontan, inventada por los doctores Fontan y Baudet en 1971, la cual consiste en la derivación de la vena cava inferior a la rama derecha de la arteria pulmonar con el fin de crear dos circulaciones, una para el retorno sistémico y otra para el flujo pulmonar ${ }^{2,17,18}$. En la actualidad, la derivación cavopulmonar superior (Glenn) es el segundo estadio quirúrgico de paliación en el corazón univentricular y no se cuenta con datos de factores pronósticos de esta cirugía en nuestro centro cardiovascular. ${ }^{19,20}$

De acuerdo con lo anterior, el objetivo del estudio fue describir las características clínicas, quirúrgicas y posquirúrgicas de pacientes con corazón univentricular atendidos en un centro de referencia cardiovascular durante su estadio de Glenn. Además, se requieren datos que permitan conocer los factores predictores de mortalidad en estos pacientes, para desarrollar pautas de manejo que permitan aumentar su sobrevida.

\section{Método}

Se realizó un estudio descriptivo, retrospectivo, en el que se incluyeron todos los pacientes con cardiopatías congénitas con fisiología univentricular que contaron con primer estadio de paliación univentricular y que consultaron a un centro de cuarto nivel de la ciudad de Medellín, Colombia, entre enero de 2012 y diciembre de 2016. Se excluyeron los pacientes que no tenían indicación de cirugía de Glenn.

A todos los pacientes remitidos con diagnóstico de ventrículo único se les realizó ecocardiografía pediátrica institucional con el fin de confirmar el tipo de anatomía univentricular presente en cada uno de ellos. En los casos en que se tenían dudas de la anatomía se llevó a cabo cateterismo cardiaco diagnóstico y resonancia cardiaca o tomografía de tórax, o ambas. Cada paciente se presentó en junta médico-quirúrgica, integrada por cardiólogos pediátricos, cirujanos cardiovasculares pediátricos, anestesiólogos cardiovasculares, intensivistas pediátricos y hemodinamistas pediátricos, quienes decidieron que cumplían criterios de corazón univentricular.

Luego fueron llevados a cirugía de primer estadio de paliación univentricular o a seguimiento clínico en caso de no requerir intervención quirúrgica temprana. El seguimiento se hizo por consulta externa en el programa de ventrículo único. Antes de la cirugía de Glenn se realizaron ecocardiografía y cateterismo cardiaco 
diagnóstico, en el cual se hizo estudio hemodinámico completo; cuando la resistencia vascular pulmonar era $>2$ unidades Wood se clasificaba como hipertensión pulmonar, y solo cuando era $>8$ unidades Wood se hicieron pruebas de reactividad pulmonar. En casos dudosos se realizó tomografía pulmonar y con estos datos se presentaron en una nueva junta médico-quirúrgica para verificar que estos pacientes podían ser llevados a cirugía de Glenn.

Para este estudio, la anatomía univentricular se dividió, posterior a su definición anatómica, en univentricular funcional derecha y univentricular funcional izquierda.

El programa de ventrículo único está a cargo de un cardiólogo pediatra y una enfermera entrenada en corazón univentricular, que tratan a los pacientes ambulatorios y siguen de manera estrecha, incluso semanal, luego del egreso hospitalario del primer estadio, hasta la realización de la cirugía de Glenn. Las madres son entrenadas para reconocer signos y síntomas de alarma y acudir al centro cardiovascular cuando presenten «banderas rojas", enseñadas en la hospitalización y en la consulta ambulatoria. Previo a la cirugía de Glenn, a los pacientes se les realizó un cateterismo cardiaco diagnóstico para presentarlo en junta médico-quirúrgica.

Posterior a la autorización del Comité de Investigación de la Clínica Cardio VID y el Comité de Ética de Investigación en Salud de la Universidad Pontificia Bolivariana, se revisaron las historias clínicas de los pacientes incluidos y se recolectaron las siguientes variables: sexo, peso $\leq 10 \mathrm{~kg}$ y presión media en la arteria pulmonar medida por cateterismo y definida por estudios previos como $>18 \mathrm{mmHg}$ en corazón univentricular $6,7,9,21$. Se definió como flujo pulmonar aumentado una relación entre el flujo pulmonar y el flujo sistémico $>2$. Se tomaron las variables de tiempo de circulación extracorpórea en la cirugía de Glenn, edad del paciente al momento de la cirugía de Glenn en días, diagnóstico anatómico determinado por ecocardiografía y complementado en caso de duda por cateterismo cardiaco, relación entre flujo pulmonar y flujo sistémico > 2 e índice de resistencia vascular pulmonar medido en unidades Wood por metro cuadrado determinado por cateterismo cardiaco y definido como $>2$. También se registró si el paciente presentaba insuficiencia de la válvula atrioventricular determinada por ecocardiograma y cateterismo, uso de terapia antihipertensiva pulmonar previo a la cirugía, tipo de intervención quirúrgica realizada con anterioridad a la cirugía de Glenn, tiempo de circulación extracorpórea en baipás cardiopulmonar $>70$ minutos (que se consideró como prolongado) y mortalidad posquirúrgica.
Los datos se procesaron en el programa SPSS versión 20.

\section{Resultados}

Durante el periodo de estudio se incluyeron 88 pacientes que acudieron remitidos a un centro de cuarto nivel en la ciudad de Medellín. De ellos, 56 (63\%) eran varones. En el total de pacientes, el promedio de edad fue de 11.4 meses, con un rango de 2 a 108 meses. Los pacientes tenían un peso promedio de $7.4 \mathrm{~kg}$ al momento de la cirugía de Glenn, variando entre 3 y $28.2 \mathrm{~kg}$, lo cual corresponde a un $93 \%$ de pacientes con peso $<10 \mathrm{~kg}$.

De acuerdo con la anatomía morfológica ventricular se decidió dividir en corazón univentricular funcional izquierdo, corazón univentricular funcional derecho y morfología indeterminada. Se encontró corazón funcional univentricular derecho en 34 pacientes (38.6\%) y morfología izquierda en 54 (61.4\%); no hubo pacientes con morfología indeterminada. En la tabla 1 se especifican los tipos de anatomía univentricular.

Se verificó en las historias clínicas la realización o no del primer estadio de paliación univentricular y se encontró que a 34 pacientes (38.6\%) se les realizó cirugía de fístula de Blalock-Taussig, a 19 (21.6\%) se les hizo cirugía de Norwood, a 9 (10.2\%) cirugía de Damus-Kaye-Stansel, 23 (26.1\%) no requirieron cirugía de primer estadio o fueron llevados a manejo hemodinámico de primer estadio sin necesidad de procedimiento quirúrgico, y a $3(3.4 \%)$ se les realizó cerclaje de la arteria pulmonar.

Se encontró que el $75 \%$ no presentó insuficiencia de la válvula atrioventricular, el $20.5 \%$ presentó insuficiencia leve, el $1.1 \%$ tuvo insuficiencia moderada y el $3.4 \%$ tuvo insuficiencia grave. En los casos de insuficiencia valvular grave, debido a la comorbilidad de los pacientes se dio manejo compasivo y no se hicieron intervenciones cardiovasculares posteriores.

De acuerdo con el cateterismo realizado antes de la cirugía de Glenn, 54 pacientes $(61,4 \%)$ presentaron presión pulmonar normal y $34(38,6 \%)$ presión pulmonar elevada del corazón univentricular cuando la resistencia vascular pulmonar superó 2 unidades Wood o presión media pulmonar $>18 \mathrm{~mm} \mathrm{Hg}$ en el cateterismo cardiaco.

En los pacientes con hipertensión pulmonar, las presiones pulmonares variaron entre 19 y $44 \mathrm{mmHg}$, con una media de $24.9 \mathrm{mmHg}$.

De todos los pacientes del estudio, 80 (90,9\%) tenían flujo pulmonar normal debido al flujo garantizado por 
Tabla 1. Anatomía del corazón univentricular en los pacientes del estudio $(n=88)$

\begin{tabular}{|l|c|}
\hline Anatomía & n (\%) \\
\hline Síndrome de ventrículo izquierdo hipoplásico & $24(25.0)$ \\
\hline Atresia tricúspide sin vasos en transposición & $20(22.7)$ \\
\hline Canal atrioventricular no balanceado & $12(13.6)$ \\
\hline Heterotaxia & $7(7.95)$ \\
\hline Atresia pulmonar con comunicación interventricular & $7(7.95)$ \\
\hline Atresia pulmonar con septum intacto & $4(4.54)$ \\
\hline $\begin{array}{l}\text { Doble salida de ventrículo único de morfología } \\
\text { derecha }\end{array}$ & $3(3.4)$ \\
\hline Doble entrada al ventrículo izquierdo & $3(3.4)$ \\
\hline Ventrículo izquierdo límite & $2(2.27)$ \\
\hline Anomalía de Ebstein grave & $2(2.27)$ \\
\hline Doble entrada al ventrículo derecho & $1(1.13)$ \\
\hline Atresia tricúspide con vasos en transposición & $1(1.13)$ \\
\hline $\begin{array}{l}\text { Doble salida de ventrículo único de morfología } \\
\text { izquierda }\end{array}$ & $1(1.13)$ \\
\hline Atresia mitral & $1(1.13)$ \\
\hline
\end{tabular}

el primer estadio de paliación univentricular y $8(9 \%)$ presentaron flujo pulmonar aumentado.

Del total de los pacientes evaluados, 41 (46\%) tenían resistencia vascular pulmonar $>2$ unidades Wood, y en ellos las resistencias vasculares pulmonares variaron entre 2.13 y 6.89 unidades Wood, con una media de 3.49 unidades Wood.

Se documentó el uso de terapia antihipertensiva pulmonar previa a la cirugía de Glenn en 26 pacientes (29.5\%). Se usó terapia para choque con medicamentos vasopresores antes de la cirugía de Glenn en 9 pacientes (18\%), en todos con milrinona. Se encontró que el tiempo promedio de perfusión fue de 94 minutos y ocurrió un fallecimiento intraquirúrgico entre los pacientes sometidos a este procedimiento. La mortalidad posquirúrgica previa al egreso hospitalario de la cirugía de Glenn fue del 18\% (16 pacientes). En la tabla 2 se muestran las características principales de los pacientes fallecidos.

\section{Discusión}

Una de las anomalías congénitas más complejas y con mayor mortalidad en el mundo es el corazón univentricular. Su tratamiento está definido, desde los años 1980, en tres estadios de paliación ${ }^{10}$. El segundo estadio es la cirugía de Glenn, que es en la cual se basa este artículo y se describen las principales características preoperatorias y operatorias, y su mortalidad.

La distribución por sexo de los pacientes en este centro cardiovascular fue de un $63 \%$ de varones, frente al $57 \%$ en otros estudios ${ }^{7}$. Se identificó la anatomía cardiovascular por medio de ecocardiografía y cateterismo cardiaco, y se encontró que el $61 \%$ tuvieron ventrículo único funcional izquierdo, mientras que otros grupos reportan frecuencias que van del $30 \%$ al $62 \% \%^{5-7,19}$.

El promedio de edad de los pacientes fue de 11.4 meses, que al compararlo con otros estudios descriptivos arroja edades promedio de 23 a 74 meses $^{6,10}$. Esto explica, además, por qué el $93 \%$ de los pacientes presentaba un peso $<10 \mathrm{~kg}$, frente al $6-19 \%$ que se reporta en otros estudios en los cuales los pacientes son mayores y tienen más peso $0^{6,19}$.

En los pacientes que acudieron a este centro cardiovascular se encontró que el $38 \%$ se presentó con presión pulmonar aumentada al llegar al segundo estadio de paliación univentricular, dato que contrasta con el porcentaje mundial, que es del $18-48 \% \%^{6,7,21}$. Con el diagnóstico de hipertensión arterial pulmonar se debe iniciar manejo de la presión pulmonar, y la primera línea, en pacientes tanto biventriculares como univentriculares, son los inhibidores de la fosfodiesterasa 5 (sildenafilo) y los antagonistas del receptor de la endotelina (bosentán); en nuestro medio se usa principalmente el sildenafilo22. De los pacientes evaluados en este estudio, se determinó que el $29 \%$ tenía manejo para hipertensión arterial pulmonar, mientras que en otros centros se reporta un 6-51\% $\%^{6-9}$. La elevación de la resistencia vascular pulmonar $>2$ unidades Wood también se ha descrito como factor de mal pronóstico ${ }^{6}$. En los pacientes de este estudio se encontró que el $46 \%$ tuvo una resistencia vascular pulmonar elevada, que concuerda con el porcentaje mundial ${ }^{6-9}$. No se realizaron pruebas de reactividad pulmonar, puesto que ninguno de nuestros pacientes superó las 8 unidades Wood, debido a la adecuada selección de pacientes antes del inicio del camino univentricular y por el correcto manejo médico en los pacientes con hipertensión pulmonar en el primer estadio.

Otro de los factores vinculados con la mortalidad es la utilización de terapia vasopresora. De los pacientes que se describieron, una quinta parte tuvo este tipo de medicación y todos recibieron milrinona. Otro de los factores de mal pronóstico para el segundo estadio de paliación univentricular es la 
Rev Colomb Cardiol. 2021;28(3)

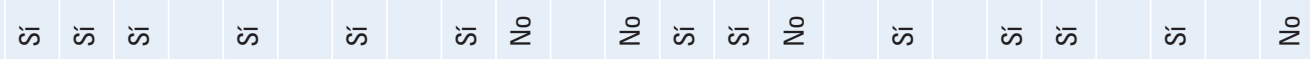

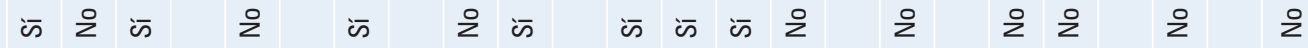

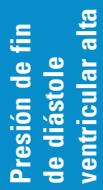

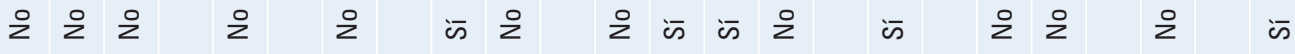

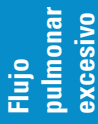

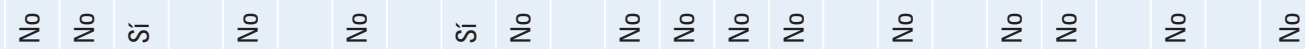

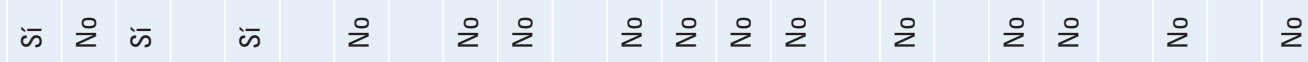

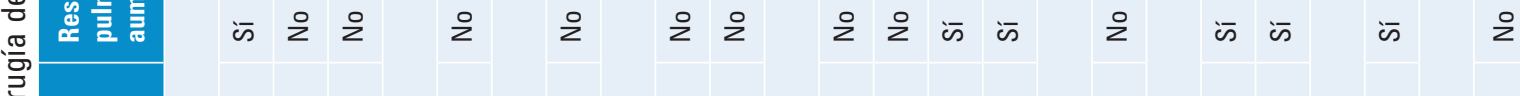
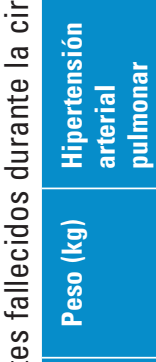

울 कㅇ z

क 을

๘ 으 ๘ 운

क क $\frac{2}{2}$ क

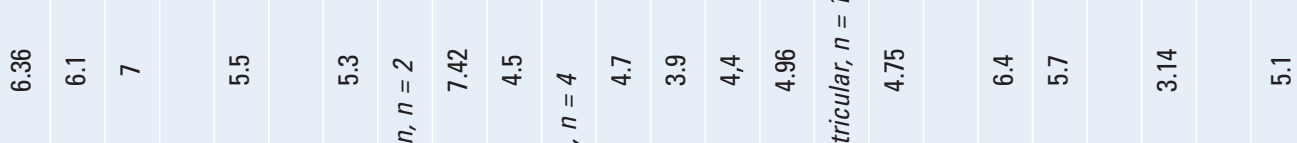
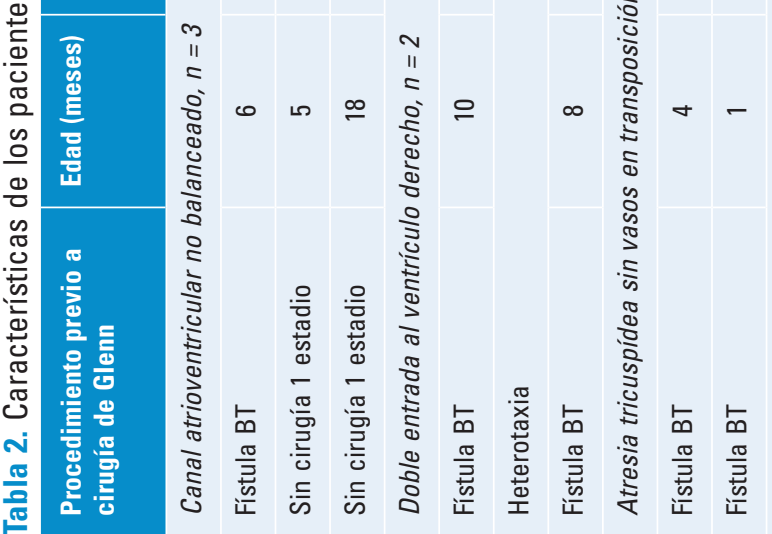

$\frac{\sqrt[0]{\frac{\pi}{2}}}{\frac{0}{2}}$

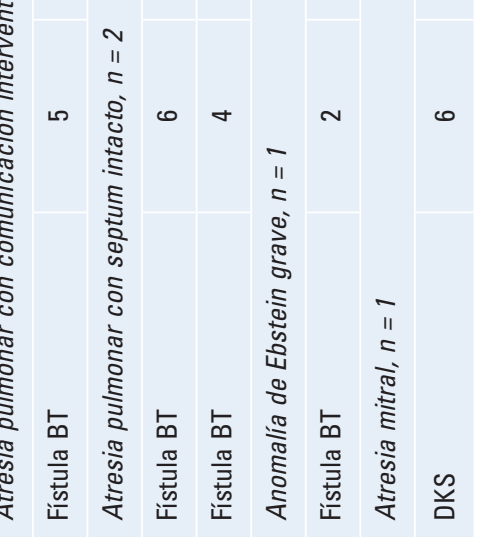


insuficiencia atrioventricular de moderada a gra$v^{6-9,21}$. Se presentó insuficiencia de la válvula atrioventricular de moderada a grave en el $25 \%$ de los pacientes (en el mundo la frecuencia es del 9-40\%), lo cual impide la realización de cirugía de Glenn para nuestro centro, lo que coincide con el promedio mundial ${ }^{6,7,21,22}$. En el presente estudio se determinó una mortalidad posoperatoria del $18 \%$ en el segundo estadio de paliación, porcentaje que está dentro de lo reportado en el mundo, que varía entre el $0,8 \%$ y el $20 \% 1,5,10,19$. En los pacientes univentriculares fallecidos se puede comprobar que todos tenían por lo menos un factor de mal pronóstico definido por estudios previos, y la mayoría tenían dos o más factores de mal pronóstico; adicionalmente, se puede evidenciar que todos ellos tenían un peso $\leq 10 \mathrm{~kg}$ y su edad era $<1$ año. La mortalidad está explicada porque los pacientes que se captaron en este estudio tenían criterios definidos de corazón univentricular claros, lo cual no está definido en otros estudios porque actualmente se prefiere operar a los pacientes con corazón univentricular de manera temprana, pues de no hacerlo su posibilidad de supervivencia es baja.

Los investigadores del estudio consideran que para el abordaje y la intervención exitosos es indispensable contar con el apoyo de un equipo multidisciplinario con experiencia en cardiopatías complejas, en el que se incluyan cardiólogos pediátricos, intensivistas cardiovasculares, anestesiólogos cardiovasculares, cirujanos cardiovasculares pediátricos y especialistas en imagen cardiaca, además de todo lo necesario para el manejo de estos pacientes desde su diagnóstico, durante la cirugía y posterior a esta, y durante su seguimiento.

El presente estudio aporta información útil para el conocimiento de este grupo de pacientes. Se hace énfasis en el diagnóstico temprano para llevar a una pronta intervención de paliación univentricular y de esta forma obtener mejores resultados en cuanto a calidad de vida y recuperación de esta.

Los datos obtenidos permiten conocer las características clínicas de estos pacientes para plantear posibles diagnósticos adicionales, como hipertensión arterial pulmonar, resistencia vascular pulmonar, choque previo a la cirugía de Glenn, insuficiencia de la válvula atrioventricular y tipo de anatomía univentricular presente, para elaborar un manejo médico óptimo.

Este estudio podría ser el punto de partida para la realización de investigaciones futuras que verifiquen los tres estadios de paliación y las características clínicas de cada uno de ellos.

\section{Agradecimientos}

A la Clínica Cardiovascular CardioVID y a los pacientes cuya información de la historia clínica permitió la realización del estudio.

\section{Financiamiento}

El presente trabajo fue financiado por la Universidad Pontificia Bolivariana y la Clínica Cardiovascular Cardio VID.

\section{Conflicto de intereses}

Los autores declaran no tener ningún conflicto de intereses.

\section{Responsabilidades éticas}

Protección de personas y animales. Los autores declaran que para esta investigación no se han realizado experimentos en seres humanos ni en animales.

Confidencialidad de los datos. Los autores declaran que han seguido los protocolos de su centro de trabajo sobre la publicación de datos de pacientes.

Derecho a la privacidad y consentimiento informado. Los autores han obtenido el consentimiento informado de los pacientes y/o sujetos referidos en el artículo. Este documento obra en poder del autor de correspondencia.

\section{Bibliografía}

1. Antelo CA, Mon HF, Pedraza C, Bencini S, Loyarte M, García R, et al. Abordaje clínico quirúrgico en el síndrome de corazón izquierdo hipoplásico. Revista Argentina de Cardiología. 2000;68:232-7.

2. Hosseinpour AR, González-Calle A, Adsuar-Gómez A. ¿Qué queremos decir con el término "univentricular»? Cir Cardiov. 2015;22:193-4.

3. Zahr RA, Kirshbom P, Kopf GS, Sainathan S, Steele MM, Elder R, et al. Half a century's experience with the superior cavopulmonary (classic Glenn) shunt. Ann Thorac Surg. 2016;101:177-82.

4. Serrano F, Caffarena JM. Cirugía del corazón univentricular en segundo estadio: técnicas y resultados. Cir Cardiov. 2008;15:351-60.

5. Clausen $\mathrm{H}$. Hypoplastic left heart syndrome. Paediatrics and Child Health. 2014:25:118-22.

6. Caspi J, Pettitt TW, Ferguson TB Jr, Stopa AR, Sandhu SK. Effects of controlled anterograde pulmonary blood flow on cardiac function after bidirectional cavopulmonary anastomosis. Ann Thorac Surg. 2003; 761917-22.

7. Alejos JC, Williams RG, Jarmakani JM, Galindo AJ, Isabel-Jones JB, Drinkwater $\mathrm{D}$, et al. Factors influencing survival in patients undergoing the bidirectional Glenn anastomosis. Am J Cardiol. 1995;75:1048-50.

8. Petrucci O, Khoury PR, Manning PB, Eghtesady P. Outcomes of the bidirectional Glenn procedure in patients less than 3 months of age. J Thorac Cardiovasc Surg. 2010;139:562-8.

9. Cnota JF, Allen KR, Colan S, Covitz W, Graham EM, Hehir DA, et al. Superior cavopulmonary anastomosis timing and outcomes in infants with single ventricle. J Thorac Cardiovasc Surg. 2013;145:1288-96.

10. Kostelka M, Hucín B, Tláskal T, Chaloupecký V, Reich O, Janousek J, et al Bidirectional Glenn followed by total cavopulmonary connection or primary total cavopulmonary connection? Eur J Cardiothorac Surg. 1997;12:177-83. 
Rev Colomb Cardiol. 2021;28(3)

11. Fernández Pineda L, Cazzaniga M, Villagrá F, Ignacio Díez Balda J, Daghero F, Herraiz Sarachaga H, et al. La operación de Glenn bidireccional en 100 casos con cardiopatías congénitas complejas: factores determinantes del resultado quirúrgico. Rev Esp Cardiol. 2001; 54:1061-74.

12. Kansy A, Brzezińska-Rajszys G, Zubrzycka M, Mirkowicz-Małek M, Maruszewski $\mathrm{P}$, Manowska $\mathrm{M}$, et al. Pulmonary artery growth in univentricular physiology patients. Kardiologia Polska. 2013;71:581-7.

13. Jalali A, Jones GF, Licht DJ, Nataraj C. Application of mathematical modeling for simulation and analysis of hypoplastic left heart syndrome (HLHS) in pre- and postsurgery conditions. BioMed Res Int. 2015;10:1-14.

14. Benson M, Martin LW, Lo C. Genetics of hypoplastic left heart syndrome. J Pediatr. 2016;173:25-31.

15. Tabbutt S, Tweddell JS, Ghanayem N. Hypoplastic left heart syndrome and other shunt-dependent single ventricles. Pediatr Crit Care Med. 2016;17:318-22

16. Jones J, Olbrych SK, Smith K, Cnota J, Habli M, Ramos-Gonzales O. Hypoplastic left heart syndrome is associated with structural and vascular placental abnormalities and leptin dysregulation. Placenta. 2015; 36:1078-86.
17. Feinstein J, Benson D, Dubin A, Cohen M, Maxey DM, Mahle W, et al. Hypoplastic left heart syndrome. Current Considerations and Expectations. 2012;59:1-42.

18. Damien J. Short and long-term outcomes for bidirectional Glenn procedure performed with and without cardiopulmonary bypass. Ann Thorac Surgery. 2012;94:164-71

19. Tanoue $\mathrm{Y}$, Kado $\mathrm{H}$, Boku $\mathrm{N}$, Tatewaki $\mathrm{H}$, Nakano $\mathrm{T}$, Fukae $\mathrm{K}$, et al Three hundred and thirty-three experiences with de bidirectional Glenn procedure in a single institute. Interact Cardiovasc Thorac Surg. 2007;6:97-101.

20. Vargas FJ, Mengo G, Gallo JP, Skerl CB, Ranzzini ME, Vazquez JC, et al. Bidirectional cavopulmonary shunt in patients with multiple risk factors. Ann Thorac Surg. 1995;60:S558-62.

21. Galie N, Humbert M, Vachiery JL, Gibbs S, Lang I, Torbicki, A et al. Guidelines for the diagnosis and treatment of pulmonary hypertension. Eur Heart J. 2016;37:67-119.

22. Damien J, Mery C, Peeler B, Kron IL, Gangemi JJ. Short and long-term outcomes for bidirectional glenn procedure performed with and with and cardiopulmonary bipass. Ann thoracic surgery. 2012; 94(1):164-70 